\title{
AN EQUICONTINUITY CONDITION IN TOPOLOGICAL DYNAMICS
}

\author{
JOHN D. BAUM ${ }^{1}$
}

It is the purpose of this paper to prove the following:

TheOREM. Let $X$ be a compact Hausdorff space, $T$ an abelian topological group, and $(X, T, \pi)$ a transformation group; then the following statements are equivalent: (I) For each index $\alpha$ of $X$ there exist a replete semigroup $P_{\alpha \beta} \subset T$ and an index $\beta$ of $X$ such that $(x, y) \in \beta$ implies $(x p, y p) \in \alpha$ for each $p \in P_{\alpha \beta}$. (II) $T$ is uniformly equicontinuous.

The reference for definitions both in the above theorem and in what follows is Topological dynamics by W. H. Gottschalk and G. A. Hedlund, Providence, 1955. It is tacitly assumed both in the statement of the theorem and in the remainder of the paper that the uniformity of $X$ induces the (Hausdorff) topology. We observe that it is obvious that (II) implies (I); the remainder of the paper is devoted to proving the converse statement. For each of the following lemmas we take the following three statements as hypotheses: (1) $X$ is a compact Hausdorff space, (2) For each index $\alpha$ of $X$ there exist an index $\beta$ of $X$ and a replete semigroup $P_{\alpha \beta} \subset T$ such that $(x, y) \in \beta$ implies $(x p, y p) \in \alpha$ for each $p \in P_{\alpha \beta}$, (3) $T$ is an abelian topological group. Full use of all these hypotheses is not made in every lemma. Throughout the paper $P_{\alpha \beta}$ will be used to denote a replete semigroup with the property mentioned in (2) above with respect to the indices $\alpha$ and $\beta$.

Lemma 1. There exists a finite partition $\left\{A_{i} \mid 1 \leqq i \leqq n\right\}$ of $X$ such that $A_{i} p \times A_{i} p \subset \alpha$ for all $p \in P_{\alpha \beta}$ and all $i, 1 \leqq i \leqq n$.

Proof. Select $\beta$ and $P_{\alpha \beta}$ according to hypothesis (2). Select $\delta$, a symmetric index of $X$, such that $\delta^{2} \subset \beta$. Since $X$ is compact there is a finite set $\left\{x_{i} \mid 1 \leqq i \leqq n\right\}$ such that $\bigcup_{i=1}^{n} x_{i} \delta=X$. Let $A_{1}=x_{1} \delta, A_{2}=x_{2} \delta$ $-x_{1} \delta, \cdots, A_{n}=x_{n} \delta-\bigcup_{i=1}^{n-1} x_{i} \delta$. Then $\left\{A_{i} \mid 1 \leqq i \leqq n\right\}$ is a partition of $X$. Let $x, y \in A_{i}$; then $x, y \in x_{i} \delta$, whence $\left(x, x_{i}\right)\left(x_{i}, y\right) \in \delta^{2} \subset \beta$ and $(x p, y p) \in \alpha$ for all $p \in P_{\alpha \beta}$. Thus $A_{i} p \times A_{i} p \subset \alpha$ for all $p \in P_{\alpha \beta}$ and all $i, 1 \leqq i \leqq n$.

Presented to the Society, January 29, 1960 under the title An equicontinuity condition for transformation groups; received by the editors January 25, 1960.

1 The author is a National Science Foundation Science Faculty Fellow. 
Lemma 2. There exists a finite set of elements $F=\left\{p_{j} \mid 1 \leqq j \leqq m\right\}$ $\subset P_{\alpha \beta}$ such that $\bigcup_{j=1}^{m} p_{j} \alpha^{4 *} \supset P_{\alpha \beta}$, where $\alpha^{*}=\left\{(p, q) \mid p, q \in P_{\alpha \beta},(x p, x q)\right.$ $\in \alpha$ for all $x \in X\}$.

Proof. Without loss of generality we may assume that $\alpha$ is symmetric, in which case $\alpha^{n}, n>0$, are also symmetric. By Lemma 1 , there exists a finite partition $\left\{A_{i} \mid 1 \leqq i \leqq n\right\}$ of $X$ such that $A_{i} p$ $\times A_{i} p \subset \alpha$ for all $p \in P_{\alpha \beta}$ and all $i, 1 \leqq i \leqq n$. Since $X$ is compact there exists a finite set $E=\left\{x_{j} \mid 1 \leqq j \leqq k\right\}$ such that $X=\bigcup_{j=1}^{k} x_{j} \alpha=E \alpha$. Thus for each $i, 1 \leqq i \leqq n$, and for each $p \in P_{\alpha \beta}$, there is some $j, 1 \leqq j \leqq k$, such that $x_{j} \alpha \cap A_{i} p \neq \varnothing$, whence $A_{i} p=x_{j}\left[\alpha \circ\left(A_{i} p \times A_{i} p\right)\right] \subset x_{j} \alpha^{2}$. Hence for each $p \in P_{\alpha \beta}$ and each $i, 1 \leqq i \leqq n$, there exists at least one $x_{j} \in E$ such that $A_{i} p \subset x_{j} \alpha^{2}$. Define for each $p \in P_{\alpha \beta}$ a class of mappings $p^{*}:\left\{A_{i}\right\} \rightarrow E$ by $p^{*}=\left\{t \mid A_{i} p \subset A_{i} t \alpha^{2}\right.$ for $\left.1 \leqq i \leqq n\right\}$. By the preceding remarks $p^{*}$ is nonvacuous for each $p \in P_{\alpha \beta}$. However, the number of mappings of $\left\{A_{i}\right\}$ into $E$ is finite (at most $k^{n}$ ), thus there exists a finite subset $F \subset P_{\alpha \beta}$ such that $\bigcup_{p \in P} p^{*}=\bigcup_{f \in F} f^{*}$. We prove $P_{\alpha \beta}=U_{f \in F} f \alpha^{4 *}$. Let $p \in P_{\alpha \beta}$. Select $t \in p^{*}$, then $t \in f^{*}$ for some $f \in F$, whence $A_{i} p \subset A_{i} t \alpha^{2}$ and $A_{i} f \subset A_{i} t \alpha^{2}$ for $1 \leqq i \leqq n$. If $x \in X$, then $x \in A_{i}$ for some $i$, whence $x p \in A_{i} p \subset A_{i} t \alpha^{2}$ and $x f \in A_{i} f \subset A_{i} t \alpha^{2}$. Hence $(x f, x p) \in\left(x p, A_{i} t\right)\left(A_{i} t, x f\right) \in \alpha^{4}$ and $x p \in x f \alpha^{4}$ for all $x \in X$. Thus $p \in f \alpha^{4 *}$ and $P_{\alpha \beta}=U_{f \in F} f \alpha^{4 *}$. Finally let $F=\left\{p_{j} \mid 1 \leqq j \leqq m\right\}$.

Lemma 3. There exists a finite set of elements, in fact $\left\{p_{j}^{-1}\right\}=F^{-1}$ $C P_{\alpha \beta}^{-1}$ (where $F^{-1}=\left\{p^{-1} \mid p \in F\right\}$ and $F$ is the set of Lemma 2) such that $\bigcup_{j=1}^{m} p_{j}^{-1} \alpha^{4 *} \supset P_{\alpha \beta}^{-1}$.

Proof. Let $p^{-1} \in P_{\alpha \beta}^{-1}$; then $p \in p_{j} \alpha^{4 *}$ for some $p_{j}$. Thus $\left(p, p_{j}\right) \in \alpha^{4 *}$ and $\left(x p, x p_{j}\right) \in \alpha^{4}$ for all $x \in X$. Therefore $\left(\left(x p^{-1} p_{j}^{-1}\right) p,\left(x p^{-1} p_{j}^{-1}\right) p_{j}\right)$ $=\left(x p_{j}^{-1}, x p^{-1}\right) \in \alpha^{4}$ for all $x \in X$, whence $\left(p_{j}^{-1}, p^{-1}\right) \in \alpha^{4 *}$ and $p^{-1}$ $\in p_{j}^{-1} \alpha^{4 *}$. Therefore $\bigcup_{j=1}^{m} p_{j}^{-1} \alpha^{4 *} \supset P_{\alpha \beta}^{-1}$.

\section{LEMMA 4. $T$ is totally bounded in its space index uniformity.}

Proof. Let $\bar{\gamma}$ be an index of the space uniformity of $T$. Select $\alpha$, an index of $X$ such that $\bar{\alpha}$ is an index of the uniformity base of the space index uniformity of $T$, i.e., $\bar{\alpha}=\{(t, s) \mid t, s \in T,(x t, x s) \in \alpha$ for all $x \in X\}$, and such that $\bar{\alpha}^{8} \subset \bar{\gamma}$. Let $G=F F^{-1}$, where $F$ is the finite set of Lemma 2, whence $G$ is finite.

Now by Lemma $2 P_{\alpha \beta} \subset F \alpha^{4 *} \subset F \bar{\alpha}^{4}$, and by Lemma $3 P_{\alpha \beta}^{-1} \subset F^{-1} \alpha^{4 *}$ $\subset F^{-1} \bar{\alpha}^{4}$. Let $p, q \in P, x \in X$, then there exists $f_{1} \in F$ such that $\left(x p, x f_{1}\right) \in \alpha^{4}$ for all $x \in X$, and in particular $\left(x p q^{-1}, x f_{1} q^{-1}\right)$ $=\left(x q^{-1} p, x q^{-1} f_{1}\right) \in \alpha^{4}$ for all $x \in X$; Also there exists $f_{2} \in F$ such that $\left(x f_{2}^{-1}, x q^{-1}\right) \in \alpha^{4}$ for all $x \in X$ and in particular $\left(x f_{1} q^{-1}, x f_{1} f_{2}^{-1}\right) \in \alpha^{4}$ for 
all $x \in X$. Consequently $\left(x p q^{-1}, x f_{1} f_{2}^{-1}\right)=\left(x p q^{-1}, x f_{1} q^{-1}\right)\left(x f_{1} q^{-1}, x f_{1} f_{2}-1\right)$ $\in \alpha^{8}$ for all $x \in X$, whence $p q^{-1} \in f_{1} f_{2}^{-1} \bar{\alpha}^{8}$ and $P_{\alpha \beta} P_{\alpha \beta}^{-1} \subset F F^{-1} \bar{\alpha}^{8}$ $=G \bar{\alpha}^{8} \subset G \bar{\gamma}$.

Since $P_{\alpha \beta}$ is a replete semigroup it follows from 6.04 (op. cit.) that $T=P_{\alpha \beta} P_{\alpha \beta}^{-1} \subset G \bar{\gamma}$, whence $T$ is totally bounded in its space index uniformity.

Proof (of the theorem). Since $X$ is compact, $X$ is totally bounded and $T$ is uniformly continuous. Thus by Ascoli's Theorem [4.38 (op. cit.) ] it follows from Lemma 4 that $T$ is uniformly equicontinuous.

It is perhaps worth mentioning that among other things, we have proved that if $X$ is a compact metric space and $T$ has the space index topology, then if $P \subset T, P$ and $P^{-1}$ are isometrically equivalent. Thus in particular, if $f$ is a homeomorphism acting in a compact metric space and $\left\{n_{j}\right\}$ is a set of integers, then $\left\{f^{n_{j}}\right\}$ and $\left\{f^{n_{i}}\right\}^{-1}=\left\{f^{-n_{j}}\right\}$ are isometrically equivalent.

Finally, the referee has kindly pointed out that with minor modififications of the proof, particularly of Lemma 4, we could prove that the following somewhat more general condition is equivalent to uniform equicontinuity of $T$ : there exists an integer $k$ such that given any index $\alpha$ of $X$, then there exists an index $\beta$ of $X$ and a subset $D_{\alpha \beta}$ of $T$ such that $T=D_{\alpha \beta}^{k} D_{\alpha \beta}^{-k}$ and $(x, y) \in \beta$ implies $(x d, y d) \in \alpha$ for all $d \in D_{\alpha \beta}$. Of course, it is here assumed that $T$ is abelian and $X$ compact Hausdorff.

Oberlin College 